\title{
Slamming load on trimaran cross section with rigid and flexible arches
}

Zhe Sun ${ }^{1}$, Yi-chen Jiang ${ }^{1,3}$, Gui-yong Zhang*1,2,3, Zhi Zong ${ }^{1,2,3}$, Jing Tang Xing ${ }^{4}$, Kamal Djidjeli ${ }^{4}$

${ }^{1}$ Liaoning Engineering Laboratory for Deep-Sea Floating Structures, School of Naval Architecture, Dalian University of Technology, Dalian, 116024, P. R. China

${ }^{2}$ State Key Laboratory of Structural Analysis for Industrial Equipment, Dalian, 116024, P. R. China

${ }^{3}$ Collaborative Innovation Center for Advanced Ship and Deep-Sea Exploration, Shanghai, 200240, P. R. China

${ }^{4}$ FSI / CED group, FEPS, University of Southampton, Southampton, SO17 1BJ, U.K.

\begin{abstract}
:
In this paper, the mixed mode function-modified MPS method was adopted to simulate the waterstructure dynamic interaction during slamming impacts on the cross deck of trimaran. The water was considered as an incompressible inviscid fluid and the "conceptual particle" model was used to enhance the stability of the intense free surface interaction during the "filling-up" process under the cross deck. The numerical model for the coupled rigid and flexible modal superposition model was derived for the dynamics of the trimaran hull with rigid and flexible arches, and the flexible arch was simplified by a beam structure. The fluid and structure solvers were coupled in a standard iterative way. The results for rigid-body arch cases obtained with the use of improved free surface condition show good improvement, in comparison to the experiment data. From the study of flexible arch cases with different flexibilities, it is found that the relatively soft structure can reduce the local pressure and slamming load.
\end{abstract}

\section{Key word:}

Trimaran; Moving Particle Semi-implicit (MPS) method; Slamming; Flexible arch; Fluid stricture interaction

\section{Introduction}

The multi-hull ships (including catamaran and trimaran) provide large deck area, which offer more spaces for both commercial and military purposes [1]. However due to the special cross deck structure, apart from the slamming on the hulls, the slamming underneath the cross deck (often referred to as "wet deck slamming" ) can cause large impulsive load and consequent whipping response of the whole ship. This could result in very serious damage of the structures. Fig. 1.1-1.2 show two typical structural failures of catamarans under slamming load. 


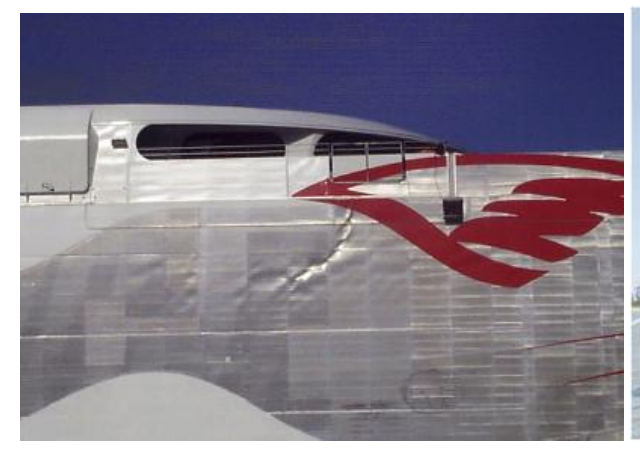

Fig. 1.1. The external plate buckling of INCAT Hull after an extreme slam event[2]

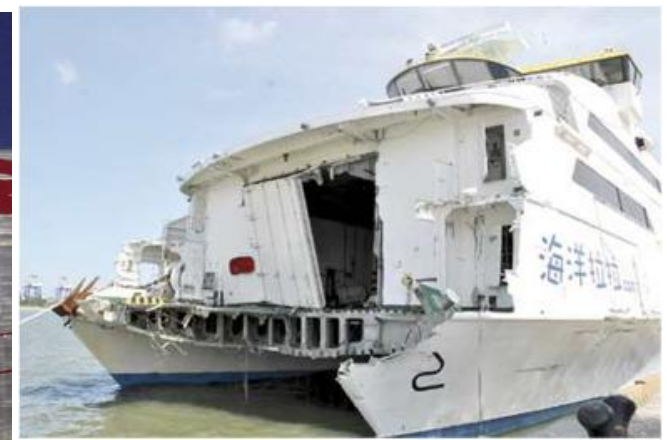

Fig. 1.2. Severe damage of Ocean LaLa following an extreme wet-deck slam event [3]

As a result, the research on the nature of slamming, predicting the magnitude of the load and finally reducing it has been an active topic of the scientific community. The full-scale measurement of load and impact pressure on ships during their operation can provide much valuable information about the features of the load under different conditions and consequently a better understanding of this complex phenomena. A number of such experiments have been successfully conducted, e.g. the series measurements of INCAT vessels by Thomas et al[4] and Amin et al[5], the slamming behavior investigation of the catamaran $H S V-2$ Swift by Jacobi et al[6]. Model-scale hydroelasticity tests have also been conducted to investigate the slamming induced global response such as whipping for catamaran $([2,7])$. On the other hand, the 2D dropping tests have been regarded as a more common and efficient way to investigate the characteristics of slamming load $([8,9])$, many of which were conducted for structure with simple geometries such as wedge ([10-12]). For multi-hulls, Whelan[13] has studies a series of 2D free falling tests for hulls with different forms. Hassoon et al.[14-16] have also conducted experiments and numerical simulations to study the performance of composite structure subject to slamming force and its failure mechanism during the slamming event.

The theoretical study of slamming load can be found at least as early as in the 1930s by von Karman[17] and Wagner[18]. Following their pioneering work, a number of models have been proposed such as similarity solutions for wedges[19], matched asymptotic expansions for small dead-rise angle impacts[20] and modified added mass method[9] etc. Moreover, the time domain boundary element method has also been successfully applied on the wedge and circular shape section dropping problems[21]. However, the difficulties of handling complex geometry and large deformation of the free surface make theoretical or potential flow based methods not as practical as the models that directly solve the Navier-Stokes equations (i.e. Computational Fluid Dynamics methods or CFD for short). Many mesh-based CFD methods have been coupled with FEM solvers for simulating the fluid structure interaction process during slamming, e.g. wave impact load on wave-piercing catamaran by commercial software StarCCM+ [3], one-way FSI (Fluid Structure Interaction) method to calculate the slam-induced bending of INCAT catamaran also using StarCCM+[7]. But the mesh-based CFD methods face their own challenges when dealing with large free surface deformation and solid boundary displacement. Also, the feature of large rigid-body displacement plus relatively small elastic deformation for the dynamics of typical marine structures makes the FEM method not necessarily time consuming for this kind of problems.

Particle methods such as MPS (Moving Particle Semi-implicit) method [22] and SPH (Smoothed Particle Hydrodynamics) are mesh-less and Lagrangian CFD methods, which means no mesh distortion and automatic flow configuration updating in time domain. These features make it 
very suitable for the problems like slamming. As a matter of fact, there have been many successful applications of particle methods for various highly non-linear fluid structure interaction problems [23-26] such as slamming (for. rigid or flexible wedge, curved surface structure etc.), sloshing etc.

In this paper, in order to investigate the flow details of slamming during the filling-up process underneath a trimaran hull and the effect of elastic arches on overall load and local pressure distribution, the 2D model that couples modified MPS method and modal superposition (rigid and flexible) method is developed. The rest of this paper is organized as follows: the fluid and structure numerical models are illustrated in Section 2; and the details of the experiment used for the validation of numerical models are given in Section 3. Section 4 provides the detailed discussion of the simulation results; and finally the conclusion is drawn in the last section.

\section{Numerical model for fluid and structure dynamics}

In this study, the modified MPS method and coupled rigid-body/elastic modes method are used for the simulation of slamming process on trimaran hull. A brief illustration of the numerical models is given in this section[23].

\subsection{Fluid solver}

\subsubsection{Modified MPS method}

Since the viscosity effect is negligible in the slamming process, the incompressible inviscid Navier-Stokes equation and continuity equation in the Langrangian frame are used in this study, as shown in Eq. (2.1.1):

$$
\begin{aligned}
& \frac{D \boldsymbol{u}}{D t}=\boldsymbol{g}-\frac{\nabla p}{\rho} \\
& \nabla \cdot \boldsymbol{u}=0
\end{aligned}
$$

where $\boldsymbol{u}, p$ and $\rho$ represent fluid velocity, pressure and density, $\boldsymbol{g}=[0,-g]$ is the vector pointing to the gravity direction.

The continuous operators are discretized on scattered particles in a weighted averaging manner as in Eq. (2.1.2)

$$
\begin{aligned}
& \nabla \phi\left(\boldsymbol{r}_{i}\right)=\frac{d_{m}}{n_{0}} \sum_{j \neq i}^{N} \frac{\phi\left(\boldsymbol{r}_{j}\right)-\phi\left(\boldsymbol{r}_{i}\right)}{r_{i j}^{2}}\left(\boldsymbol{r}_{j}-\boldsymbol{r}_{i}\right) W\left(r_{i j}\right) \\
& \nabla^{2} \phi\left(\boldsymbol{r}_{i}\right)=\frac{2 d_{m}}{n_{0} \lambda} \sum_{j \neq i}^{N}\left[\phi\left(\boldsymbol{r}_{j}\right)-\phi\left(\boldsymbol{r}_{i}\right)\right] W\left(r_{i j}\right) \\
& \nabla \cdot \boldsymbol{\Phi}\left(\boldsymbol{r}_{i}\right)=\frac{d_{m}}{n_{0}} \sum_{j \neq i}^{N} \frac{\left(\boldsymbol{\Phi}\left(\boldsymbol{r}_{j}\right)-\boldsymbol{\Phi}\left(\boldsymbol{r}_{i}\right)\right) \cdot\left(\boldsymbol{r}_{j}-\boldsymbol{r}_{i}\right)}{r_{i j}^{2}} W\left(r_{i j}\right)
\end{aligned}
$$

in which $\phi$ and $\boldsymbol{\Phi}$ represent arbitrary scalar and vector variables, $d_{m}$ is the dimension of the problems, and $N$ is the number of particles within the radius $r_{e}$ of the compact support domain for the current particle that is being discretized. The weight function $W\left(r_{i j}\right)$, particle density $n$ and parameter $\lambda$ are defined in Eq. (2.1.3)-(2.1.4), respectively.

$$
W\left(r_{i j}\right)=\left\{\begin{array}{cc}
\frac{r_{e}}{r_{i j}}-1 & 0 \leq r_{i j} \leq r_{e} \\
0 & r_{i j} \geq r_{e}
\end{array}\right.
$$




$$
\begin{gathered}
n=\sum_{j \neq i}^{N} W\left(r_{i j}\right) \\
\lambda=\frac{\sum_{j \neq i}^{N} W\left(r_{i j}\right) r_{i j}^{2}}{\sum_{j \neq i}^{N} W\left(r_{i j}\right)}
\end{gathered}
$$

The incompressibility condition is enforced by managing the particle distribution to be as even as possible, which involves three steps: firstly, the flow is advanced to the intermediate state by just the inertia and gravity without considering the pressure; secondly, a pressure Poisson equation is derived based on the continuity equation; lastly, the position and velocity of the fluid are updated by the newly computed pressure. In the modified MPS model[23], in order to achieve the balance between even particle distribution and small unphysical pressure fluctuation, the following pressure Poisson equation was derived:

$$
\nabla^{2} p_{n+1}=\frac{\rho \nabla \cdot \boldsymbol{u}_{*}}{\Delta t}+\alpha \rho \frac{n_{0}-n_{n}}{n_{0} \Delta t^{2}}
$$

where $n_{0}$ and $n_{n}$ are particle density for initial and $n^{\text {th }}$ time step, respectively. The coefficient $\alpha$ is defined in Eq. (2.1.7).

$$
\alpha= \begin{cases}\left|\frac{n_{0}-n_{n}}{n_{0}}\right|+\Delta t\left|\nabla \cdot \boldsymbol{u}_{n}\right| & \left(n_{0}-n_{n}\right) \nabla \cdot \boldsymbol{u}_{n} \geq 0 \\ \left|\frac{n_{0}-n_{n}}{n_{0}}\right| & \left(n_{0}-n_{n}\right) \nabla \cdot \boldsymbol{u}_{n} \leq 0\end{cases}
$$

The form of $\alpha$ requires no special calibration according to different problems, which makes it a more robust model compared to other similar forms[27, 28].

Several other techniques were also developed in modified MPS model to improve the accuracy, stability and efficiency, which include particle shifting, new efficient way of performing neighbor particle searching, the use of Neumann type boundary condition, the consistent way of updating the intermediate velocity of the solid boundary particles and virtual particle outside the solid boundary. More implementation details could be found in ref[23].

On the free surface, the solving of the Poisson equation Eq. (2.1.6) requires the identification of free surface particles and imposing zero pressure boundary condition on it. In modified MPS model, the simplified version of the technique used by Koh[29] was developed. However, when solving the problems which involve the intensive free surface particle interaction such as the filling up of fluid under the arch of trimaran, the sudden change of the particle status from free surface to inner particles may cause instability in the simulation. To overcome this problem, an improved free surface condition proposed by Chen et al[30] are incorporated with the modified MPS method developed by the authors. More details are given in the following section.

\subsubsection{Improved free surface condition}

In the area where particles suddenly change their status from free surface to inner particles, the Laplacian operator of the nearby particles will be affected dramatically and consequently cause instability or breakdown of the computation under the traditional zero-pressure free surface boundary condition, especially when these changes occur to a group of particles with more than just one or two particles. As shown in Fig. 2.1, most of the free surface particles (marked as red) around particle $A$ change into inner particles (marked as blue circles and dots) after the transition from $t_{n}$ to $t_{n+1}$ time step. In the traditional zero-pressure boundary condition, which means the coefficients associated to free surface particles will be eliminated in the discretization of Laplacian operator for particle $A$, this sudden change in time step $t_{n+1}$ will cause a corresponding sudden change of the 
Laplacian coefficient structure of particle $A$ (equivalent to suddenly add several particles in the neighbor of particle $A$ ). This means the resultant pressure will be dramatically increased or even cause local explosion if this increase is too large.
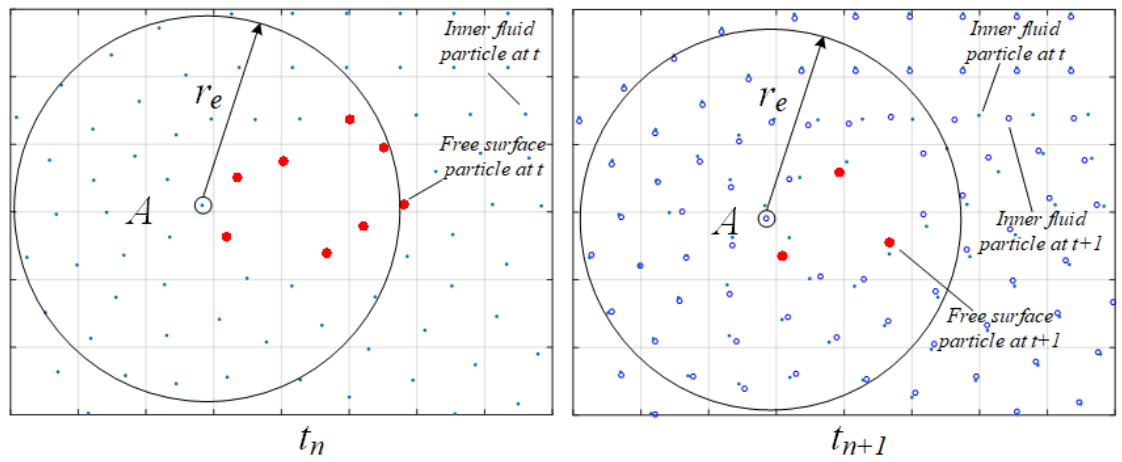

Fig. 2.1 Schematic diagram of dramatic change of free surface status

In order to tackle this issue in this study, the technique so-called conceptual particle[30] model was added to the modified MPS method. The implementation details are illustrated briefly as follows:

For free surface particles (whose particle density is smaller than $n_{0}$ ), instead of imposing zeropressure condition, the Poisson equation will also be solved for inner fluid particles. The so-called conceptual particles with zero pressure are added in the support domain of the concerned particle to make sure its particle density becomes $n_{0}$. As a result, the zero pressure condition is imposed on the conceptual particles instead of the free surface particles. And this means the change from free surface to inner particles will not cause the sudden change of coefficient in Laplacian discretization as mentioned before. Then by substituting both real and conceptual particles into the Laplacian discretization (i.e. Eqn. (2.1.2)-(2)), the coefficient corresponding to the concerned particle will be $n_{0}\left(2 d / \lambda n_{0}\right)=2 d / \lambda$. Since the pressure of the conceptual particles is zero, the coefficient of the other real particles will not be affected and the existing of the conceptual particles will not be explicitly represented in the equation (i.e. new degrees of freedom) as well. This means that the positions of the conceptual particles will not be needed.

One final issue with this technique is that if the particle density of a free surface particle is larger than $n_{0}$ (i.e. when there are particles that are very close to this free surface particle), the conceptual particle should at least make the final particle density larger than that value (otherwise, imposing the value of $n_{0}$ by the conceptual particles actually reduce the coefficient for the concerned particle, which means reducing the resultant pressure). In this case, the target particle density for the concerned particle is calculated by Eqn. (2.1.8).

$$
\tilde{n}_{i, n+1}=n_{0}+\sum_{j \neq i}^{N_{r}}\left[w\left(r_{i j}\right)-w\left(l_{0}\right)\right]
$$

in which $N_{r}$ is the number of particles inside the circle with radius of $l_{0}$ centered by the concerned particle. Finally, the Poisson equation for all fluid particles is discretized by Eqn. (2.1.9).

$$
\left\{\begin{array}{l}
\frac{2 d}{\lambda n_{0}} \widehat{n}_{i, n+1} p_{i, n+1}-\frac{2 d}{\lambda n_{0}} \sum_{j \neq i}^{M}\left(w\left(r_{i j}\right) p_{j, n+1}\right)=\frac{\rho\left\langle\nabla \cdot \boldsymbol{u}_{*}\right\rangle_{i}}{\Delta t}+\alpha \rho \frac{n_{0}-n_{i, n}}{n_{0} \Delta t^{2}} \\
\widehat{n}_{i, n+1}=\max \left(n_{i, n+1}, \tilde{n}_{i, n+1}\right)
\end{array}\right.
$$

where $n_{i, n}$ and $n_{i, n+1}$ mean the particle density for $i^{\text {th }}$ particle at $t=t_{n}$ and $t=t_{n+1}$ respectively.

\subsection{Structure solver}

The flexibility of the arches (where the highest load occurs) will be considered in this study. 
The coupled rigid-body and elastic modes model developed by the authors [24] are applied here. A brief derivation is given below. The model is based on Lagrangian equation as given in Eqn. (2.2.1):

$$
\frac{d}{d t}\left(\frac{\partial T}{\partial \dot{q}_{j}}\right)+\frac{\partial U}{\partial q_{j}}-\frac{\partial T}{\partial q_{j}}=Q_{j} \quad j=1,2,3, \ldots
$$

where $T$ and $U$ are the kinetic and potential energy of the structure system, respectively, $q_{j}$ is the general coordinates for any rigid/flexible mode, and $Q_{j}$ represents the non-conservative forces corresponding to $j^{\text {th }}$ coordinate. The specific forms of $T, U, q_{j}$ and $Q_{j}$ for the trimaran hull structure with rigid/flexible arches are given later in this section.

The global fixed $X-O-Y$ system and local body-attached $s-O-w$ system are used for the description of the trimaran hull structure as shown in Fig. 2.2. Two identical arches are represented by beam model with fixed ends on both sides (the origin of the $s-O-w$ system is placed at the center of the beam). The dynamics of the trimaran hull could be represented by the deflection of the beam $\eta$ and the position of the gravity center (i.e. $\left.O_{R}\right) \mathbf{X}_{R}=\left[X_{R}, Y_{R}\right]^{T}$.

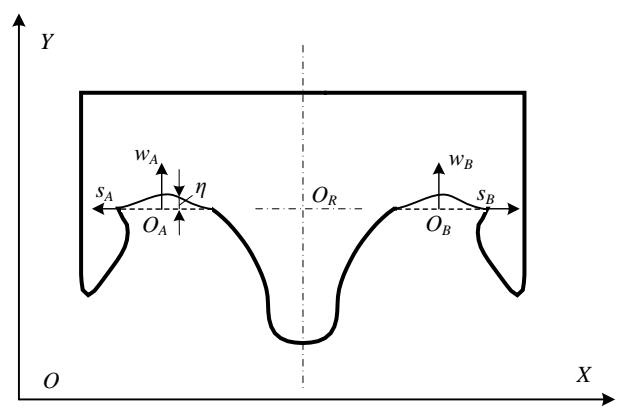

Fig. 2.2 Sketch of the model for trimaran hull with rigid/flexible arches

The position of any point on the flexible arches can be calculated by Eqn. (2.2.2):

$$
\mathbf{X}=\mathbf{X}_{R}+\xi
$$

in which $\xi=[s, \eta]^{T}$. Considering the symmetry feature of the configuration and the consequent dynamic response during the slamming process, the $X$ direction translation and rotation of the body are restricted in the model, i.e. $X_{R}=$ const. . The elastic deformation $\eta$ is represented by the modal superposition approach. More specifically, the spatial and time dimensions of $\eta$ are treated separated by a set of mode function $\boldsymbol{\varphi}=\left[\varphi_{1}, \varphi_{2}, \varphi_{3}, \ldots\right]^{T}$ and the corresponding general coordinates $\mathbf{q}=\left[q_{1}, q_{2}, q_{3}, \ldots\right]^{T}$. As a result, the deflection is formulated as Eqn. (2.2.3):

$$
\eta=\boldsymbol{\varphi}^{T} \mathbf{q}
$$

The following orthogonal conditions in Eqn. (2.2.4)-(2.2.5) are satisfied by the mode function $\varphi$.

$$
\begin{gathered}
\int_{s_{1}}^{s_{2}} \boldsymbol{\varphi} \rho_{l} \boldsymbol{\varphi}^{T} d s=\mathbf{I} \quad \mathbf{I} \text { is } \text { unit matrix } \\
\int_{s_{1}}^{s_{2}} \frac{d^{2} \boldsymbol{\varphi}}{d s^{2}} E I \frac{d^{2} \boldsymbol{\varphi}^{T}}{d s^{2}} d s=\mathbf{\Lambda}, \quad \mathbf{\Lambda}=\operatorname{diag}\left(\omega_{i}^{2}\right) \quad i=1,2,3, \ldots
\end{gathered}
$$

The fixed-fixed type beam is used to represent the flexible arch, and the mode shape function 
is given in Eqn. (2.2.6):

$$
\varphi_{i}=\frac{1}{\sqrt{M_{F}}}\left[\cosh \left(\beta_{i} x\right)-\cos \left(\beta_{i} x\right)-\sigma_{i}\left(\sinh \left(\beta_{i} x\right)-\sin \left(\beta_{i} x\right)\right)\right], \quad \sigma_{i}=\frac{\cos \left(\beta_{i} L\right)-\cosh \left(\beta_{i} L\right)}{\sin \left(\beta_{i} L\right)-\sinh \left(\beta_{i} L\right)}
$$

where $L$ is the length of the flexible arch, $x$ is the distance from the $-L / 2$ of the axis in $s-O-w$ system to any point on the beam in Fig. 2.2. The parameter $\beta_{i}$ corresponding to the first three modes, are given by: $\beta_{1}=4.7300407446, \quad \beta_{2}=7.8532046242, \quad \beta_{3}=10.9956078382$.

The kinetic energy $T$ and potential energy $U$ of the whole trimaran hull system could be calculated by adding the contribution from flexible arches and the rest rigid-body parts together as in Eqn. (2.2.7)-(2.2.8):

$$
\begin{aligned}
T & =\frac{1}{2}\left(\int_{s_{A 1}}^{s_{A 2}} \dot{\mathbf{X}}^{T} \rho_{l} \dot{\mathbf{X}} d s+\int_{s_{B 1}}^{s_{B 2}} \dot{\mathbf{X}}^{T} \rho_{l} \dot{\mathbf{X}} d s\right)+\frac{1}{2} M_{R} \dot{Y}_{R}{ }^{2} \\
& =\frac{1}{2}\left(\int_{s_{A 1}}^{s_{A 2}}\left(\dot{\mathbf{X}}_{R}+\dot{\xi}\right)^{T} \rho_{l}\left(\dot{\mathbf{X}}_{R}+\dot{\xi}\right) d s+\int_{s_{B 1}}^{s_{B 2}}\left(\dot{\mathbf{X}}_{R}+\dot{\xi}\right)^{T} \rho_{l}\left(\dot{\mathbf{X}}_{R}+\dot{\xi}\right) d s\right)+\frac{1}{2} M_{R} \dot{Y}_{R}{ }^{2} \\
& =\frac{1}{2}\left[\left(M_{R}+2 M_{F}\right) \dot{Y}_{R}{ }^{2}+\dot{\mathbf{q}}_{A}{ }^{T} \dot{\mathbf{q}}_{A}+\dot{\mathbf{q}}_{B}{ }^{T} \dot{\mathbf{q}}_{B}+2 \dot{Y}_{R}\left(\dot{\mathbf{q}}_{A}{ }^{T}+\dot{\mathbf{q}}_{B}{ }^{T}\right) \boldsymbol{\Psi}_{0}\right] \\
U & =\frac{1}{2}\left(\int_{s_{A 1}}^{s_{A 2}} \frac{d^{2} \eta_{A}}{d s^{2}} E I \frac{d^{2} \eta_{A}}{d s^{2}} d s+\int_{s_{B 1}}^{s_{B 2}} \frac{d^{2} \eta_{B}}{d s^{2}} E I \frac{d^{2} \eta_{B}}{d s^{2}} d s\right)+\left(M_{R}+2 M_{F}\right) g Y_{R} \\
& =\frac{1}{2}\left(\mathbf{q}_{A}{ }^{T}\left(\int_{s_{1}}^{s_{2}} \frac{d^{2} \boldsymbol{\varphi}}{d s^{2}} E I \frac{d^{2} \boldsymbol{\varphi}^{T}}{d s^{2}} d s\right) \mathbf{q}_{A}+\mathbf{q}_{B}{ }^{T}\left(\int_{s_{1}}^{s_{2}} \frac{d^{2} \boldsymbol{\varphi}}{d s^{2}} E I \frac{d^{2} \boldsymbol{\varphi}^{T}}{d s^{2}} d s\right) \mathbf{q}_{B}\right)+\left(M_{R}+2 M_{F}\right) g Y_{R}(2.2) \\
& =\frac{1}{2}\left(\mathbf{q}_{A}{ }^{T} \boldsymbol{\Lambda} \mathbf{q}_{A}+\mathbf{q}_{B}{ }^{T} \boldsymbol{\Lambda} \mathbf{q}_{B}\right)+\left(M_{R}+2 M_{F}\right) g Y_{R}
\end{aligned}
$$

where $M_{R}$ and $M_{F}$ represent the mass of the rigid-body and flexible parts respectively. The subscript $A$ or $B$ indicates that the corresponding value is for beam $A$ or $B, E I$ is the stiffness, and $\rho$ is the line density of the beam.

In Eqn. (2.2.7), $\psi_{0}$ is defined as:

$$
\boldsymbol{\psi}_{0}=\left[\psi_{01}, \psi_{02}, \psi_{03}, \ldots\right]^{T}=\int_{s_{1}}^{s_{2}} \boldsymbol{\varphi} \rho_{l} d s
$$

After substituting Eqn. (2.2.7)-(2.2.8) into Eqn. (2.2.1), the governing equations for the dynamics of the trimaran hull with flexible arches are derived in Eqn. (2.2.10)

$$
\begin{aligned}
& \left(M_{R}+2 M_{F}\right) \ddot{Y}_{R}+\boldsymbol{\psi}_{0}{ }^{T}\left(\ddot{\mathbf{q}}_{A}+\ddot{\mathbf{q}}_{B}\right)+\left(M_{R}+2 M_{F}\right) g=Q_{Y_{R}} \\
& \ddot{Y}_{R} \boldsymbol{\psi}_{0}+\ddot{\mathbf{q}}_{A}+\Lambda \mathbf{q}_{A}=\mathbf{Q}_{q_{A}} \\
& \ddot{Y}_{R} \boldsymbol{\psi}_{0}+\ddot{\mathbf{q}}_{B}+\Lambda \mathbf{q}_{B}=\mathbf{Q}_{q_{B}}
\end{aligned}
$$

The non-conservative forces for rigid and flexible variables are given in Eqn. (2.2.11):

$$
\begin{aligned}
& Q_{Y_{R b}}=\llbracket p n_{y} d l \\
& \mathbf{Q}_{q_{A}}=\int_{A} p\left(\mathbf{n} \cdot \mathbf{e}_{\eta}\right) \boldsymbol{\phi} d s \\
& \mathbf{Q}_{q_{B}}=\int_{B} p\left(\mathbf{n} \cdot \mathbf{e}_{\eta}\right) \boldsymbol{\varphi} d s
\end{aligned}
$$

where $\mathbf{n}=\left[n_{x}, n_{y}\right]$ is the normal vector of the structure surface pointing towards the inside of the solid 
boundary, and $\mathbf{e}_{\eta}$ is the unit vector of O- $\eta$ axis. Based on the symmetry feature of the structure, the model could be further simplified as follows:

$$
\begin{aligned}
& \left(M_{R}+2 M_{F}\right) \ddot{Y}_{R}+2 \boldsymbol{\psi}_{0}{ }^{T} \ddot{\mathbf{q}}+\left(M_{R}+2 M_{F}\right) g=Q_{Y_{R}} \\
& \ddot{Y}_{R} \boldsymbol{\psi}_{0}+\ddot{\mathbf{q}}+\boldsymbol{\Lambda} \widehat{\mathbf{q}}=\widehat{\mathbf{Q}}_{q}
\end{aligned}
$$

where $\hat{\mathbf{q}}$ and $\hat{\mathbf{Q}}_{q}$ are defined in Eqn. (2.2.13) under the assumption that $\mathbf{q}_{A}=\mathbf{q}_{B}$.

$$
\begin{aligned}
& \widehat{\mathbf{q}}=\frac{1}{2}\left(\mathbf{q}_{A}+\mathbf{q}_{B}\right) \\
& \widehat{\mathbf{Q}}_{q}=\frac{1}{2}\left(\mathbf{Q}_{q_{A}}+\mathbf{Q}_{q_{B}}\right)
\end{aligned}
$$

The governing equation for structure, i.e. Eqn. (2.2.12) is first discretized in time domain by Newmark method[31], and then the roots for the resultant equations are solved by Newton method.

For the rigid-body case, the structure model reduces to the following equation:

$$
M_{R} \ddot{Y}_{R}=Q_{Y_{R}}-M_{R} g
$$

For the fluid and structure interaction process, the standard iterative approach is adopted until certain criterion is met. The detailed information can be found in ref[24] and will not be listed here for simplicity reason.

\section{Experiment setup}

The experiment reported in ref [32] is used for the validation of the numerical model in this study. The basic set-up information is briefly illustrated here.

As shown in Fig. 3.1, the 4.52kg trimaran hull made of 5mm thickness PMMA (Poly Methyl MethAcrylate) was placed on top of a tank. The tank is filled with $0.623 \mathrm{~m}$ depth of water, and the distance from the tip of the main hull to the water surface is $h=0.147 \mathrm{~m}$. According to the dynamics of free falling object (i.e. $\sqrt{2 g h}=V$ ), its water entry velocity is $V=1.7 \mathrm{~m} / \mathrm{s}$. The other dimensions of the tank and the trimaran hull are shown in Fig.3.1-3.2. Both pressure and acceleration are monitored in this experiment. More specially, from the bottom of the main hull up to underneath the arches, totally 5 pressure sensors are located as shown in Fig. 3.2 (along the $o-x$ axis). They are only placed on one side of the trimaran and the sampling frequency is $1000 \mathrm{~Hz}$. Three acceleration sensors are placed along the tip of the trimaran main hull to check if a good posture is maintained during the impact process; and their arrangement are shown in Fig. 3.1 (along $o-y$ axis).

For the purpose of examining the uncertainty of the slamming process, 10 trials of the dropping tests are used for the analysis. The Savitzky-Golay filter (with polynomial order and frame size chosen to be 3 and 11) is used to eliminate the high frequency noise from the original pressure and acceleration signals.

In order to investigate the free surface deformation during the filling-up underneath the arches, a laser source and high-speed camera were placed under and in front of the tank respectively. Due to the symmetric property of the flow, only half of the field was captured and recorded. The laser sheet is located at the mid-span of the specimen with the thickness of $1 \mathrm{~mm}$. The spatial resolution of the camera is $1280 \times 800$ pixels, and the acquisition frequency is $200 \mathrm{~Hz}$. 

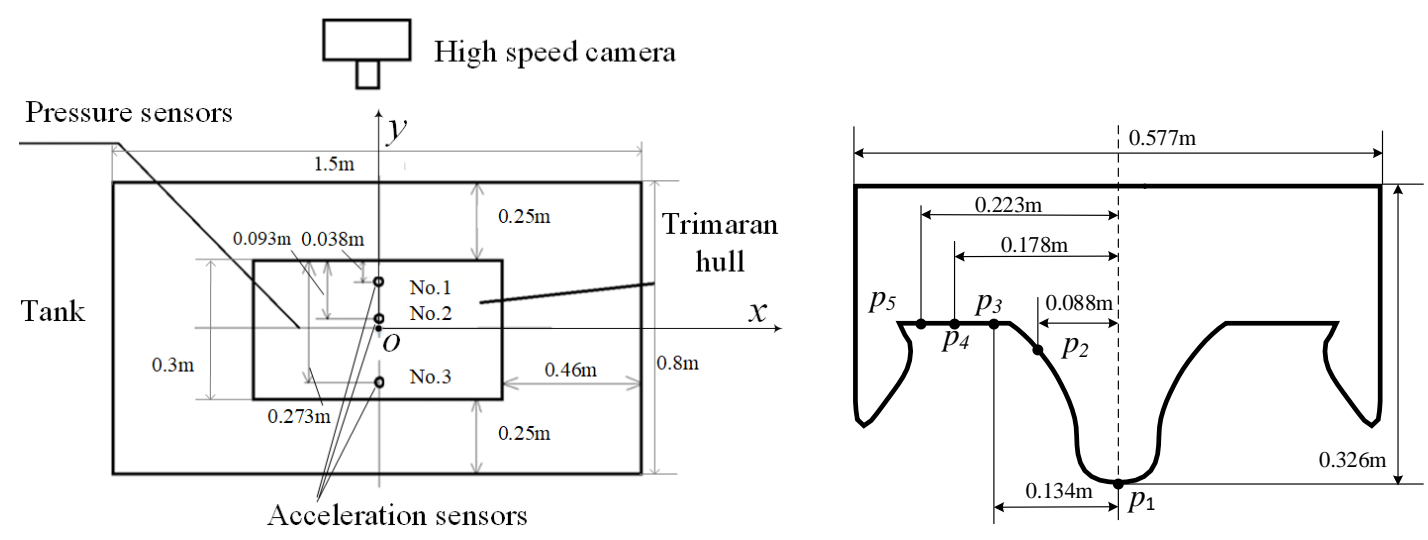

Fig. 3.1 Sketch of the experiment set-up

Fig. 3.2 Sketch of the trimaran cross section and pressure sensor layout

\section{Result and discussion}

\subsection{Rigid-body case}

In this section, the implementation details and results of the rigid-body case are discussed. The initial particle distance and time interval are chosen to be $0.01 \mathrm{~m}$ and be $0.0005 \mathrm{~s}$ respectively, and the size of time step is controlled by CFL (Courant-Friedrich-Levy) condition. The size of the computational domain is set to be the same as the experiment, i.e. $1.5 \mathrm{~m} \times 0.623 \mathrm{~m}$. Totally 10856 particles are used for the discretization, in which 9536 particles are used for fluid domain. Since this is a $2 \mathrm{D}$ computation, the computational mass is chosen to be the value corresponding to $1 \mathrm{~m}$ thick model i.e. $4.52 / 0.3=15.067 \mathrm{~kg}$. The dynamics of the trimaran hull is computed by Eqn. (2.2.13). 



Fig. 4.1 Comparison of experimental and numerical acceleration results $((a)-(c)$ corresponding to

No.1 -3 in Fig. 3.1)

Fig. 4.1 shows comparison of experimental and numerical results for the acceleration time histories. The acceleration results from three sensors show good consistency, which prove that the specimen has not tilted during the slamming process and therefore the $2 \mathrm{D}$ flow assumption is reasonable. By cross checking the time histories with the images from the camera, the instant when the tip of the main hull enters into water was identified and set to be $t=0$ in the following analysis. The small fluctuation of the signals before $t=0$ could be due to the air cushion effect. The experimental results include the mean value, one/two times of the standard deviations and envelop lines. As shown in Fig. 4.1, the result from the model with new free surface condition performs better than the original modified MPS method [14] in the sense of predicting the value and time for both initial and mean peak. In the result of original modified MPS result (blue dash line), there is a local peak at $\mathrm{t}=0.8 \mathrm{~s}$, which is caused by the sudden change of free surface distribution and consequently the abrupt increase of pressure as explained in section 2.1.2. This unphysical change of the particle and pressure distribution also results in the advancing of main peak. The result from the model with improved free surface condition proves that this problem has been successfully solved by the introducing this modification. 

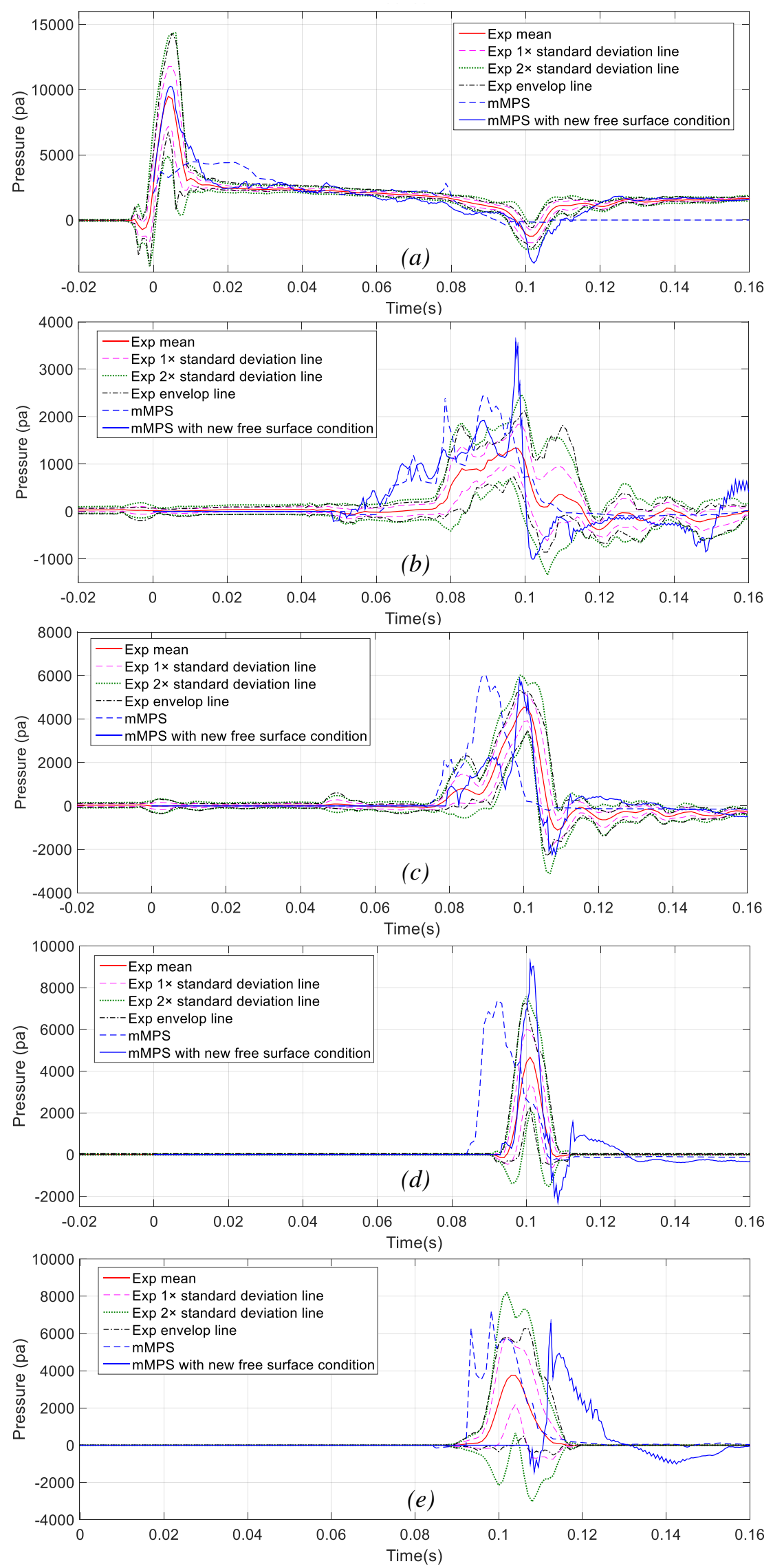

Fig. 4.2 Comparison of the pressure time histories between experimental and numerical results ((a)-(e) corresponds to $p_{1}-p_{5}$ in Fig. 3.2) 
Experiment results


Fig. 4.3 Comparison of the pressure contour (from numerical results) and free surface profiles (from experimental results)

The pressure time histories at the positions $p_{1}-p_{5}$ as marked in Fig.3.2 are shown in Fig. 4.2. 
The significant improvement of the pressure prediction can be seen in Fig. $4.2(a)$, where both the time and value of the positive and negative peaks are captured reasonably well compared with the original modified MPS model. It is also worth noticing in Fig. $4.2(a)$ that there will be a negative pressure local peak at the tip of the main hull when the slamming to the arch occurs. The main features of the pressure history (peak position and negative value after the peak in Fig. $4.2(c)-(d)$ ) around the middle of the arches are successfully captured. The value of the peaks for both numerical results are slightly higher than the mean values (closer to the upper bound of 2 times standard deviation lines), this could be due to the fact that in the experiment, the flow is not completely restricted along the direction of the main hull tip line (the tank is longer than the trimaran hull in this direction), this $3 \mathrm{D}$ effect tends to reduce the pressure on the hull, which cannot be captured in the 2D simulation. The major discrepancy occurs at $p_{5}$ (Fig. $4.2(e)$ ), where the pressure time history computed by the model with new free surface boundary condition shows roughly $0.01 \mathrm{~s}$ of delay.

The pressure contour and free surface profiles of the numerical and experimental results are compared in Fig. 4.3. The good symmetry and smoothness of the pressure field prove the feasibility of modified MPS method for slamming simulation. It is worth to mention that along the o- $y$ axis (in Fig. 3.1), the trimaran hull is shorter than the tank, and therefore the undisturbed water level in Fig. 4.3 actually shows the status of the surrounding water around the trimaran hull. For the area underneath the trimaran arches, the water filling-up process in the numerical prediction is generally consistent with the experimental result. By cross-checking with the Fig. 4.1, the maximum acceleration occurs when the region underneath the arches are completely filled with water (at around $0.985 \mathrm{~s}$ ) as corrected predicted by the numerical simulation. However the flow separation around the main hull of the trimaran is not clearly observed in the experiment.

\subsection{Flexible arch case}

The arches are the places where the maximum load for the whole trimaran hull would occur. In this section, the effect of flexible arches on the load and overall dynamics of the trimaran hull are investigated. The material property, thickness and circular frequencies (first three orders) of the flexible arches are given in Table 1. It is worth mentioning that the stiffness (i.e. EI) and mass of the arches for "Soft 1" and "Soft 2" cases are the same, hence the resulting arch deformation and overall dynamics of the trimaran hull are the same (according to the formation of the structure solver). However the stresses of the arches are different and this difference is discussed later in this session.

Table 1. Parameters of the flexible arches used in the numerical simulation

\begin{tabular}{c|c|c|c|c|c|c}
\hline Name & $\begin{array}{c}\text { Density } \rho_{\mathrm{s}} \\
\left(\mathrm{kg} / \mathrm{m}^{3}\right)\end{array}$ & $\begin{array}{c}\text { Young's modulus } \\
E(\mathrm{Gpa})\end{array}$ & $\begin{array}{c}\text { Thickness } \\
t_{\mathrm{h}}(\mathrm{mm})\end{array}$ & $\omega_{1}(\mathrm{rad} / \mathrm{s})$ & $\omega_{2}(\mathrm{rad} / \mathrm{s})$ & $\omega_{3}(\mathrm{rad} / \mathrm{s})$ \\
\hline Soft 1 & 1190 & 3.25 & 0.7 & 508.4265 & 1401.4972 & 2747.4948 \\
\hline Soft 2 & $1190 / 2$ & $3.25 / 8$ & 1.4 & 508.4265 & 1401.4972 & 2747.4948 \\
\hline Stiff & 1190 & 3.25 & 1.4 & 1016.8530 & 2802.9943 & 5494.9896 \\
\hline
\end{tabular}

The configuration and discretization of the computational domain are the same as that in the rigid-body case. Moreover, the Nyquist-Shannon sampling theorem is satisfied since the highest vibration frequency is smaller than half of the computational sampling rate (i.e. $2 \pi / 0.0005=$ $12566.3706 \mathrm{rad} / \mathrm{s}$ ), which guarantees that the dynamic features of the system can be fully captured by the simulation. 




Fig. 4.4 The acceleration time history of rigid-body and flexible-arch cases

Fig. 4.4 shows comparison of acceleration between rigid-body and flexible arch cases. The lines of rigid-body and flexible arches almost coincide with each other before the impact on the arches (at about 0.08s, as shown in Fig. 4.3). After the instant when the arches are in contact with water, the flexibility of the arches starts to significantly affect the dynamics of the acceleration (which can be seen from the load result in Fig. 4.5 as well). Generally, apart from the obvious vibrations that are roughly consistent with the frequencies of the corresponding first order natural frequency $(0.02 \mathrm{~s}$ and $0.05 \mathrm{~s}$ for stiff and soft cases respectively), the increase of flexibility will postpone the time of peak and reduce its value. This means that relatively soft arches could ease the dynamic impact of slamming for the whole structure. Another important feature for the flexible arches cases is that the negative peak has the same order of magnitude as its positive counterpart, which indicates that the flexible vibration causes large negative pressure under the arches (which is shown in Fig. 4.8). However as explained in Section 4.1 (rigid-body cases), since the flow along the tip of the hull in 3D flow cannot be captured, the pressure tends to be slightly higher in 2D flow. The same reasoning is also applicable to flexible cases, i.e. the absolute value of the negative pressure may be actually smaller in the 3D flow. This means the positive and negative peaks for flexible arches could be smaller in 3D situations, which is even better in the sense of reducing dynamic slamming impact. But this has to be verified by $3 \mathrm{D}$ experiment or simulation in the future.

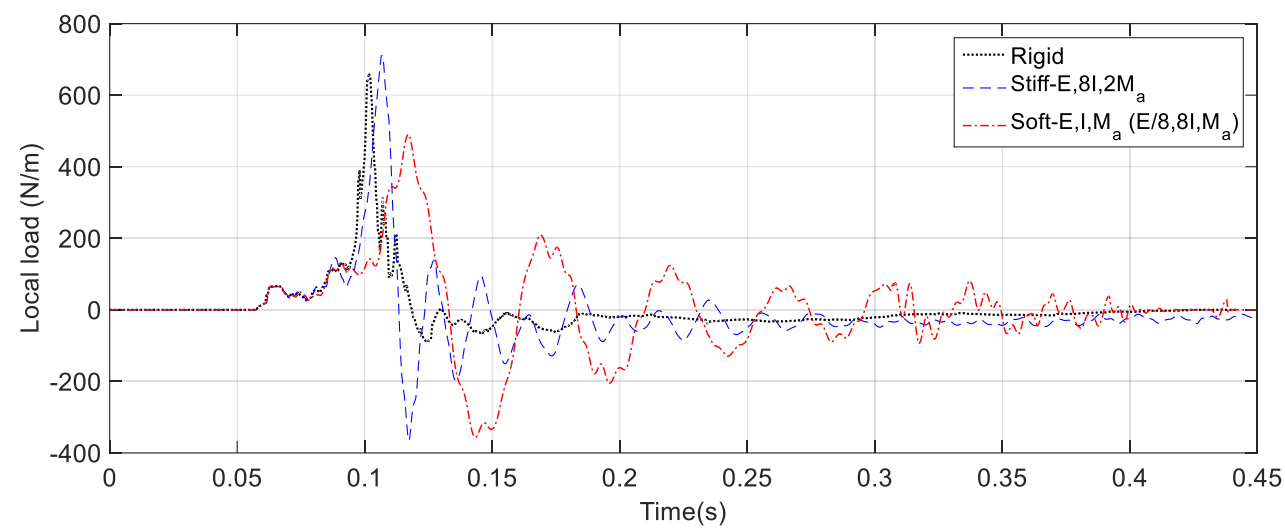

Fig. 4.5 The load time history of rigid-body and flexible-arch cases

Fig. 4.5 shows the time histories of load under the arches for rigid and flexible cases. The trends of the load are very consistent with the acceleration result in Fig. 4.4, which confirms that 
the load applied on the arches are the dominant factor that affect the dynamics of the trimaran hull. The average loads over the arches for a given time of period are summarized in Table 2. The second column shows the time average load during the first positive peaks for different cases, which indicates that soft arches, compared to stiff and rigid case, can reduce the load by about $6 \%$. In the third column of Table 2, compared to the rigid body case, the time average load from the instant when water touching the arches to $0.4 \mathrm{~s}$ (when the load for all cases tend to zero) are reduced by roughly $16 \%$ and $36 \%$ (absolute value) for the two flexible cases, respectively. The comparison between stiff and soft cases show that the soft arch tends to perform better in the sense of reducing the peak and average value during the slamming event (i.e. the first peak), while the stiff arch would give a lower average load for a longer period after the slamming event.

Table 2. Load on the arches

\begin{tabular}{c|c|c}
\hline Name & $\begin{array}{c}\text { Average load of the first } \\
\text { positive peak }(\mathrm{N})\end{array}$ & $\begin{array}{c}\text { Average load from touching } \\
\text { water to } 0.4 \mathrm{~s}(\mathrm{~N})\end{array}$ \\
\hline Soft 1 and Soft 2 & 151.6 & 10.3 \\
\hline Stiff & 159.9 & -7.9 \\
\hline Rigid & 161.7 & 12.3 \\
\hline
\end{tabular}

The time histories of the arch deflection at the middle point for soft and stiff cases are shown in Fig. 4.6. The maximum deflection of the soft case is about 5 times larger than that of the stiff one. The frequency of the oscillation is roughly consistent with the first order natural frequency of the arches, respectively.

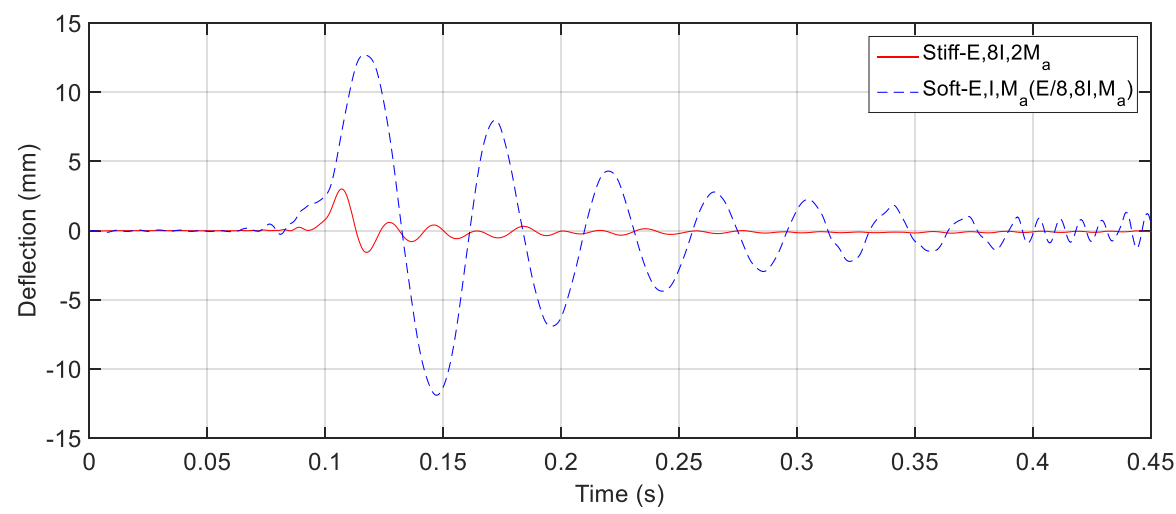

Fig. 4.6 Deflection time histories at the middle of the arch with different stiffness

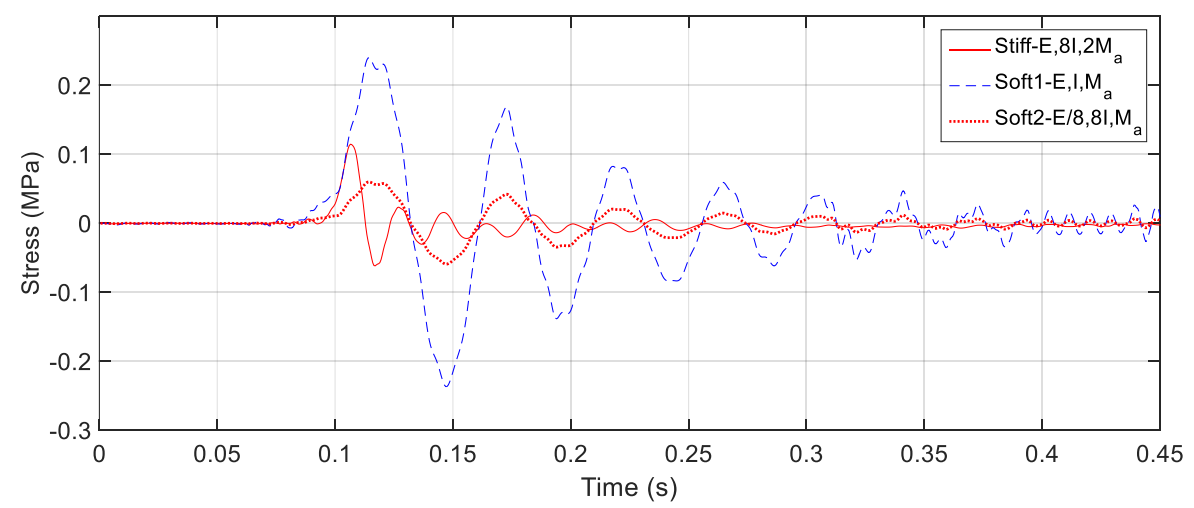

Fig. 4.7 Stress time histories at the middle of the arch with different stiffness 

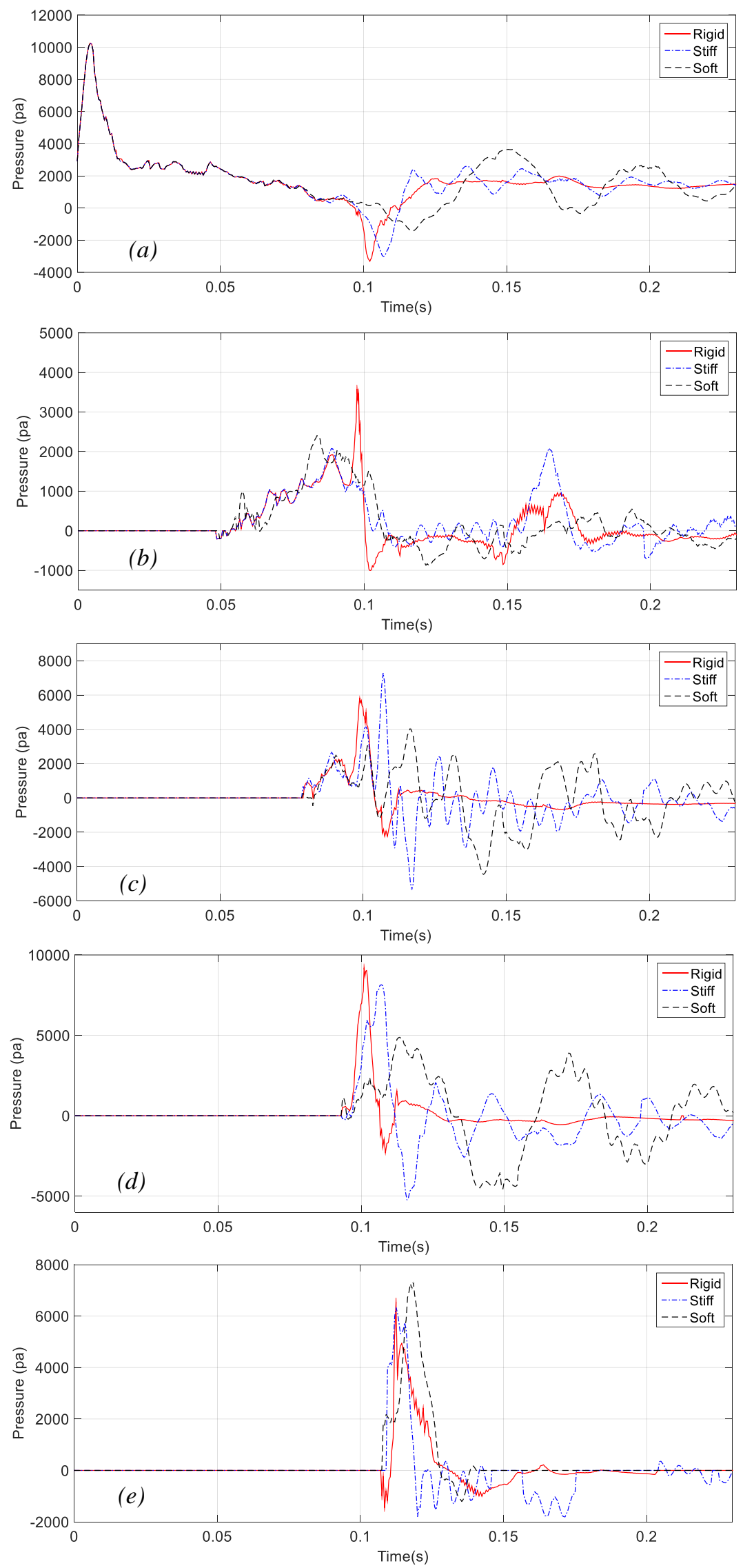

Fig. 4.8 Comparison of the pressure time histories between rigid-body and flexible arches cases ((a)-(e) correspond to $p_{1-} p_{5}$ in Fig. 3.2) 
As for the stresses of the arch, the case (i.e. Soft 1 in Table 1) with smaller Young's modulus gives much smaller stresses compared to the case with the same stiffness (i.e. Soft 2 in Table 1), though their deformation and overall dynamics are the same. This indicates that the usage of a relatively soft material can ease the slamming load (by allowing a limited deformation) without causing the dramatic increase of stress level.

The above discussion about load and stress suggests that by adding a relatively soft buffer layer of structure (with properly chosen material properties such as Young's modulus and density) on top of the arch area, the slamming load could be effectively eased without negatively affecting the original structure integrity. Also the usage of material with small Young's modulus can help to control the stress of the buffer layer in a relatively low level.

The pressure time histories at $p_{1}-p_{5}$ for flexible arches cases are shown in Fig. 4.6. The major difference between rigid-body and flexible arches cases occur on the middle of the arch, i.e. Fig. 4.6 $(c)$ and $(d)$. The oscillation of the flexible arches causes corresponding fluctuation of pressure, and the order of magnitude for positive and negative peaks in Fig. $4.6(c)$ and $(d)$ are approximately the same. Moreover, the peak pressures for the soft arch case is relatively lower (especially the middle of the arch $p_{4}$ in Fig. $4.6(d)$ ) than the rigid-body and stiff arch cases. This means a flexible layer of structure with proper choice of stiffness can reduce the slamming pressure, which is consistent with the findings in Fig. 4.4. This is an aspect worth investigating further in the future both numerically and experimentally.

The pressure distribution at the instants of the first positive and negative acceleration peak for each case are shown in Fig. 4.7. The pressure field shows good symmetry and smoothness. The stiff arch tends to generate lower pressure than the soft arch at the corresponding instants when negative acceleration peaks occur. The slight flow separation around the main hull can also be observed in the flexible arch cases. 

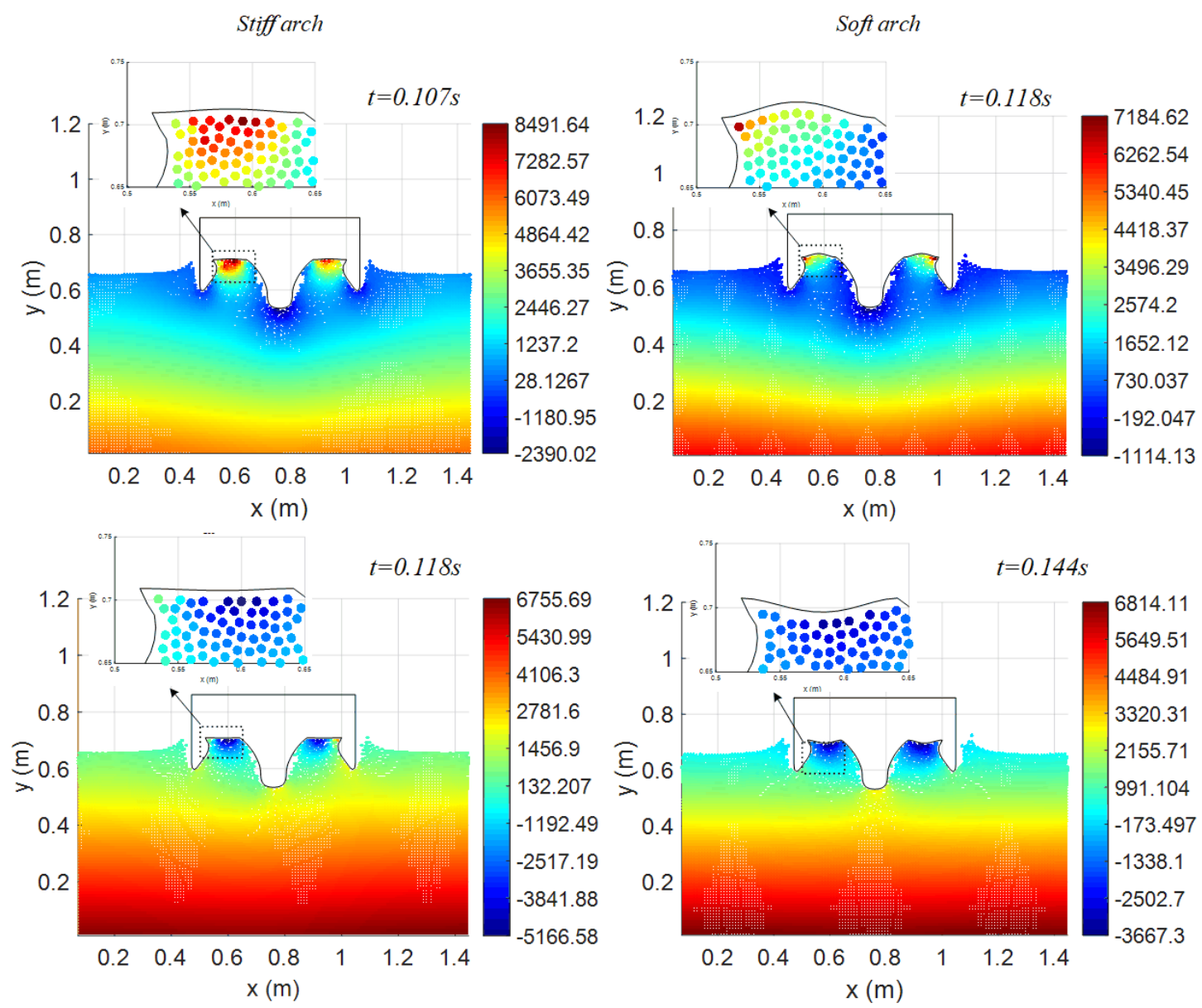

Fig. 4.9 Pressure contour and arch deflections for different stiffness cases

\section{Conclusion}

The slamming load and pressure distribution was investigated numerically in this paper. For the fluid part, the so-called "conceptual particle" model was used in the modified MPS method to improve its performance for the flow with intensive free surface deformation and interaction. Both rigid-body and flexible arch cases were simulated. The coupled rigid and flexible modal superposition model was derived to calculate the dynamics of the trimaran hull with flexible arches. The fluid and structure interaction model was validated against the experimental results and relatively good agreement was obtained, which show the feasibility of using the developed model to simulate this type of problems.

It is found that the increase of arch flexibility would postpone the time of maximum impact load/pressure under the arch, and also reduce their peak values significantly. This indicates that by adding a relatively soft buffer layer of structures on top of the original structure with properly chosen stiffness and material properties, the impact force of slamming underneath the arch can be potentially eased without significantly affecting the local strength of the original structure. This needs to be further studied numerically or experimentally in $3 \mathrm{D}$ in the future. 


\section{Acknowledgement:}

This work is supported by the National Natural Science Foundation of China (No. 51579042, 51809035, and 51639003), Fundamental Research Funds for the Central Universities (No. DUT17RC(3)017 and DUT2017TB05), Joint Found for Equipment Pre Research and China Shipbuilding Industry Corporation (6141B04010103), High-tech ship research project of Ministry of Industry and Information Technology of China (No. 2017-614) and the Supercomputing Center of Dalian University of Technology, to which the authors are most grateful.

\section{Reference:}

[1] T. Coppola and M. Mandarino, "The Design of Trimaran Ships: General Review and Practical Structural Analysis," 2001.

[2] J. Lavroff, M. R. Davis, D. S. Holloway, and G. Thomas, "Wave slamming loads on wave-piercer catamarans operating at high-speed determined by hydro-elastic segmented model experiments," Marine Structures, vol. 33, pp. 120-142, 2013.

[3] J. Lavroff, M. R. Davis, D. S. Holloway, G. A. Thomas, and J. J. McVicar, "Wave impact loads on wave-piercing catamarans," Ocean Engineering, vol. 131, pp. 263-271, 2017.

[4] G. Thomas, M. R. Davis, D. S. Holloway, N. Watson, and T. Roberts, "Slamming response of a large high-speed wave-piercer catamaran," SNAME Marine Tech J, vol. 40, p. 15, 2003.

[5] W. Amin, M. R. Davis, G. Thomas, and D. S. Holloway, "Slamming quasi-static analysis of an incat 98-m high-speed wave piercing catamaran," presented at the Proceedings of the international conference for innovation in high speed marine vehicles, Fremantle, Australia, 2009.

[6] G. Jacobi, G. Thomas, M. R. Davis, and G. Davidson, "An insight into the slamming behaviour of large high-speed catamarans through full-scale measurements," Journal of Marine Science and Technology, vol. 19, pp. 15-32, 2013.

[7] J. McVicar, J. Lavroff, M. R. Davis, and G. Thomas, "Fluid-structure interaction simulation of slam-induced bending in large high-speed wave-piercing catamarans," Journal of Fluids and Structures, vol. 82, pp. 35-58, 2018.

[8] A. Swidan, G. Thomas, D. Ranmuthugala, W. Amin, I. Penesis, T. Allen, et al., "Experimental drop test investigation into wetdeck slamming loads on a generic catamaran hullform," Ocean Engineering, vol. 117, pp. 143-153, 2016.

[9] M. R. Davis and J. R. Whelan, "Computation of wet deck bow slam loads for catamaran arched cross sections," Ocean Engineering, vol. 34, pp. 2265-2276, 2007.

[10] R. Panciroli, S. Abrate, and G. Minak, "Dynamic response of flexible wedges entering the water," Composite Structures, vol. 99, pp. 163-171, 2013.

[11] R. Panciroli and M. Porfiri, "Analysis of hydroelastic slamming through particle image velocimetry," Journal of Sound and Vibration, vol. 347, pp. 63-78, 2015.

[12] M. Jalalisendi, A. Shams, R. Panciroli, and M. Porfiri, "Experimental reconstruction of threedimensional hydrodynamic loading in water entry problems through particle image velocimetry," Experiments in Fluids, vol. 56, 2015.

[13] J. R. Whelan, "Wetdeck Slamming of High Speed Catamarans With a Centre bow," PhD, 
Univesrsity of Tasmania, Tasmania, Australia, 2004.

[14] O. H. Hassoon, M. Tarfaoui, and A. E. Moumen, "Progressive damage modeling in laminate composites under slamming impact water for naval applications," Composite Structures, vol. 167, pp. 178-190, 2017.

[15] O. H. Hassoon, M. Tarfaoui, A. E. M. Alaoui, and A. E. Moumen, "Experimental and numerical investigation on the dynamic response of sandwich composite panels under hydrodynamic slamming loads," Composite Structures, pp. 297-307, 2017.

[16] F. Erchiqui, M. Souli, T. Kanit, A. Imad, B. Aziz, and A. E. Moumen, "Characterization of Polymeric Membranes Under Large Deformations Using Fluid-Structure Coupling," International Journal of Applied Mechanics, vol. 07, 2015.

[17] T. von Karman, "The Impact on a Sea Plane Floats During Landing," Washington, USA1929.

[18] H. Wagner, "Uber Stoss-und Gleitvorgange an der Oberflache von Flussigkeiten," Zeitschrift fur Angewandte Mathematik und Mechanik, vol. 12, pp. 193-215.

[19] Z. N. Dobrovol'skaya, "On some problems of similarity flow of fluid with a free surface," Journal of Fluid Mechanics, vol. 36, pp. 805-829, 1969.

[20] R. Cointe, "Two dimensional water-solid impact," ASME Journal of Offshore Mechanics and Arctic Engineering Analysis with Boundary Elements, vol. 111, 1989.

[21] H. Sun, "A Boundary Element Method Applied to Strongly Nonlinear Wave-Body Interaction Problems," PhD thesis, Norwegian University of Science and Technology, 2007.

[22] S. Koshizuka, H. Tamako, and Y. Oka, "A particle method for incompressible viscous flow with fluid fragmentation," Computational Fluid Dynamic Journal, vol. 4, pp. 29-46, 1995.

[23] Z. Sun, K. Djidjeli, J. T. Xing, and c. Fai, "Modified MPS method for the 2D fluid structure interaction problem with free surface," Computers \& Fluids, vol. 122, pp. 47-65, 2015.

[24] Z. Sun, K. Djidjeli, J. T. Xing, and C. Fai, "Coupled MPS-modal superposition method for 2D nonlinear fluid-structure interaction problems with free surface," Journal of Fluids and Structures, vol. 61, pp. 295-323, 2016.

[25] Z. Sun, K. Djidjeli, and J. T. Xing, "The weak coupling between MPS and BEM for wave structure interaction simulation," Engineering Analysis with Boundary Elements, vol. 82, pp. 111-118, 2017.

[26] Y. Zhang and D. Wan, "MPS-FEM coupled method for sloshing flows in an elastic tank," Ocean Engineering, 2017.

[27] M. Kondo and S. Koshizuka, "Improvement of stability in moving particle semi-implicit method," International Journal for Numerical Methods in Fluids, vol. 65, pp. 638-654, 2011.

[28] B. H. Lee, J. C. Park, M. H. Kim, and S. C. Hwang, "Step-by-step improvement of MPS method in simulating violent free-surface motions and impact-loads," Computer Methods in Applied Mechanics and Engineering, vol. 200, pp. 1113-1125, 2011.

[29] C. G. Koh, M. Gao, and C. Luo, "A new particle method for simulation of incompressible free surface flow problems," International Journal for Numerical Methods in Engineering, vol. 89, pp. 1582-1604, 2012.

[30] X. Chen, G. Xi, and Z.-G. Sun, "Improving stability of MPS method by a computational scheme based on conceptual particles," Computer Methods in Applied Mechanics and Engineering, vol. 278, pp. 254-271, 2014.

[31] N. M. Newmark, "A Method of Computation for Structural Dynamics," Journal of the Engineering Mechanics Division, vol. 85, pp. 67-94, 1959. 
[32] Z. Sun, Y.-f. Sun, J. Wu, Y. Jiang, Z. Zong, and T. Sun, "Experimental and numerical study of slamming problem for a trimaran hull(submitted)," Journal of Offshore Mechanics and Arctic Engineering, 2019. 